Journal of Environmental Sciences (JES)

Institute of Environmental Studies and Research, Ain Shams University

Hussein Nourhan et al.

\title{
PATTERNS OF ANTIMICROBIAL USE AND PREVALENCE OF ANTIBIOTIC RESISTANT BACTERIA \\ STUDY AT AIN-SHAMS UNIVERSITY \\ SPECIALIZED HOSPITAL (ASUSH)
}

\author{
Nourhan Hussein ${ }^{(1)}$; Wafaa Kh. Zaki ${ }^{(2)}$ \\ and Gehan M. Fahmy ${ }^{(3)}$
}

1) Ain Shams University Specialized Hospital 2) Professor of Medical Microbiology and Immunology, Faculty of Medicine Ain Shams University 3) Professor of clinical microbiology, Infection Control Consultant Ain Shams Specialized Hospital.

\begin{abstract}
Introduction: Antibiotics are prescribed for treating bacterial infections. However, Excessive use of antibiotics leads to the development and spread of multidrug resistant bacteria, currently regarded as a global public health crisis threatening our ability to treat common infectious diseases, resulting in prolonged illness, disability and death.

Aim of the work: To analyze the use and prescribing patterns of antimicrobials by physicians at Ain-Shams University Specialized Hospital (ASUSH) for inpatients among different infections and to determine the prevalence of different types of bacterial resistance among hospital infections. Subjects and methods: A cross- sectional study was conducted at (ASUSH). A total of 339 microorganisms isolated from several samples types were identified and studied using VITEK® 2 system. Physicians' attitudes and knowledge were assessed using a questionnaire about antibiotic resistance problem.
\end{abstract}

Results: Among (339) bacterial isolates Klebsiella pneumoniae was the most common isolate $n=92(27.1 \%)$, followed by E-coli $n=60(17.7 \%)$, Staph coag -ve (SCONs) $n=43(12.7 \%)$ and Methecillin resistant staphylococcus aureas (MRSA), Pseudomonas aeruginosa and Acinetobacter baumanni n=33 (9.7\%)

Vol.(50); Iss.(1); No.(1); Jan.2021

ISSN $1110-0826$ 
for each. In this study, the majority of physicians had good knowledge about the antibiotic resistance meaning and the magnitude of that problem, with a positive attitude towards prescribing antibiotics.

Conclusion: Strategies for control of resistant bacteria should consider variations among sample types, wards, and antibiotic resistance variability. There is a need to specifically address staff training and communication procedures for infection prevention and control with respect to drug resistant bacteria.

Keywords: antibiotic policy, Multi Drug Resistance, physicians' knowledge and attitude, Antibiotic Susceptibility.

\section{INTRODUCTION}

There is no doubt that antimicrobials have played a revolutionary role in healthcare systems worldwide. From the discovery of the first antibiotics in the 1930s and1940s, these medicines have saved countless lives through reducing morbidity and mortality rates for a number of infectious diseases that were once a principal cause of death. (Nichol., et al ., 2018).

Antibiotics have not only saved patients' lives, they have played a pivotal role in achieving major advances in medicine and surgery. They have successfully prevented or treated infections that can occur in patients who have chronic diseases such as diabetes, end-stage renal disease, or rheumatoid arthritis; or who have had complex surgeries such as organ transplants, joint replacements, or cardiac surgery.(Gould and Bal., 2013 ; Atif, et al ., 2017).

New resistance mechanisms are emerging and spreading globally, threatening our ability to treat common infectious diseases. (Tyers and Wright, 2019; WHO, 2018). It leads to increased morbidity and mortality, increased hospital stay, and costly and toxic alternatives. However, while 
Journal of Environmental Sciences (JES)

Institute of Environmental Studies and Research, Ain Shams University

Hussein Nourhan et al.

resistance rates continue to rise, the rate of antibiotic discovery has dropped substantially. (Laxminarayan, 2014; Hernando-Amado, et al., 2017).

Antimicrobial resistance (AMR) happens when microorganisms (such as bacteria) change when they are exposed to antimicrobial drugs they became less sensitive to the drug and sometimes completely insensitive to the antibiotic. As a result, the medicines become ineffective and infections persist in the body, increasing the risk of spread to others (WHO, 2018).

A number of factors are thought to be associated with this rapidly developing AMR bacteria but inappropriate consumption of antimicrobial (either prescribing antimicrobials when not required or prescribing a broadspectrum agent when a narrower spectrum agent would be adequate) is still the main determinant of AMR ( Atif., et al ., 2017).

Globally, between 20 to $50 \%$ of antimicrobial consumption is inappropriate, causing significant impact on the quality of care, cost of therapy and incidence of adverse drug (Centers for Disease Control and Prevention, 2013). Inappropriate consumption of antimicrobial is not the only reason for antibiotic resistance, there are many other reasons leading to this crisis like the overuse of antibiotics \& the extensive agricultural and veterinary use of it. (Ventola, CL. 2015).

Inappropriate attitudes and lack of knowledge towards the use of antibiotics, patients' demands, lack of culture and selling antibiotics without prescription are also some of the most common contributors towards 
Journal of Environmental Sciences (JES)

Institute of Environmental Studies and Research, Ain Shams University

Hussein Nourhan et al.

antibiotic resistance and considered as a high-risk factor contributing to the rise of AMR cases. (Zulu, et al., 2020)

As a result, the WHO recommends continuous surveillance of antibiotic use as a strategy for improving antibiotic use among patients and also for controlling antibiotic resistance and minimizing patient harm, so accurate information on the use of antibiotics are crucial to address the problem of antibiotic overuse and resistance. (Labi, et al ., 2018).

We aimed to analyze the use and prescription patterns of antimicrobials by physicians at (ASUSH) for inpatients with different infections and to determine the prevalence of different types of bacterial resistance among hospital infections

\section{SUBJECT AND METHODS}

\section{Patient and sample characteristics}

Records from August 2018 to January 2019 from inpatients with MDR infections attending ASUSH were analyzed. A total number of 339 MDRpositive samples were identified. Demographic data (sex, age group) is summarized in Table (1). They were isolates from pus, urine, blood, respiratory and cerebrospinal fluid (CSF) samples; sample type information and Bacterial species identified for each sample type are summarized in Table (2). 
Journal of Environmental Sciences (JES)

Institute of Environmental Studies and Research, Ain Shams University

Hussein Nourhan et al.

\section{Identification and Antibiotic susceptibility testing}

The identification was carried out by the automated VITEK ${ }^{\circledR} 2$ system ((bioMérieux. Marcy l'Etoile, France) which used for species identification and susceptibility testing of the bacteria, which accommodate the colorimetric reagent cards that are incubated and interpreted automatically.

\section{Physicians' survey}

A questionnaire was distributed to (90) physicians at ASUSH from different departments, positions and with different years of experience to determine their knowledge and understanding of MDR infections and the procedures in place to identify and contain these infections in their institution.

\section{STATISTICAL METHODS}

IBM SPSS statistics (V. 25.0, IBM Corp., USA, 2017-2018) was used for data analysis. Date were expressed as both number and percentage for categorized data. The following tests were done:

- Descriptive statistics using Cross-tabulation including count and absolute percentage.

- Chi-square test to study the association between each two variables or comparison between two independent groups as regards the categorized data. 
Journal of Environmental Sciences (JES)

Institute of Environmental Studies and Research, Ain Shams University

Hussein Nourhan et al.

\section{RESULTS}

\section{Sample type}

In this study a total number of 339 isolates of MDR bacterial infection were isolated mainly from males $(n=210(61.9 \%))$ while females accounted for $(n=129(38.1 \%))$. The distribution of those resistant pathogens mainly found in age range (51-70 years) with (51.6\%), then from (31-50 years) with $(22.7 \%)$, which is demonstrated in table (1).

\section{Bacterial species}

Bacterial species distribution of the 339 MDR isolates and sample distribution is shown in Table (2). The total number of isolated pathogens were (339) among which Klebsiella was the most common isolate $n=92$ (27.1\%), followed by E-coli $n=60(17.7 \%)$, Staph coag - ve (SCONs) $n=43$ (12.7\%) and MRSA, Pseudomonas and Acinetobacter $n=33$ (9.7\%) for each.

Klebsiella and E_coli were frequently isolated from urine samples $32.14 \%$ and $29.7 \%$ of all isolated microorganism from urine sample respectively.

\section{Antibiotic resistance profiles}

The resistance and sensitivity profile for each microorganism in table (3) and table (4) revealed that Antibiotic resistance profiles were not uniform across bacterial species or sample types; these factors should be considered for informing antibiotic stewardship programs within the hospital. As expected, there were overall high levels of susceptibility to carbapenems. E.coli showed the lowest resistance pattern to most antibiotics unlike 
Journal of Environmental Sciences (JES)

Institute of Environmental Studies and Research, Ain Shams University

Hussein Nourhan et al.

klebsiella which was the Most frequent isolate in our study showed moderate resistance to Meropenem (51.9\%) and Amikacin (53\%) and was highly resistant to Ceftriaxone (96.5\%) followed by Ceftazidim (95.8\%) and Levofloxacin(71.4\%). Table (3)

None of MRSA nor SCONs were resistant to linezolid nor vancomycin. However, MRSA showed higher sensitivity against teicoplanin, tri/sulpha and erythromycin than SCONs. Table (4)

\section{Physicians' knowledge}

When they were asked about the meaning of antibiotic resistance and the magnitude of this problem the majority (85.6\%) Knew the meaning and felt that it's a very important problem

Overuse of broad spectrum antibiotics considered to be the most Important factor that lead to antibiotic resistant (100\%) followed by Widespread use of antibiotic (98.9\%) and inappropriate use of antibiotics $(82.2 \%)$. The least important factor was companies promote antibiotic use \& inadequate hand washing $(36.7 \%)$.

Physicians thought that prolonged Treatment (74\%) and increased cost (68\%) were the most frequent complications related to antibiotic resistance and Death is the least complication (7\%).Table (5)

Physicians thought that Educational program to physicians considered to be the most useful factor in containing the problem $(78 \%)$ followed by culture prior to antibiotic choice $(70 \%) \&$ Antibiotic cycling the least useful factor $(44 \%)$ as summarized in table(5). 


\section{Physicians' practice}

The highly prescribed Antibiotic (empiric antibiotic) were Meropenem (26.7\%), Amp/sulb (17.8), levofloxacin (15.6\%), Ceftriaxone (11.1\%), Ceftazidime (7.8\%), Ciprofloxacin (5.6\%), Amikacin (5.6\%), Dalacin (4.4\%), Tazocin (3.3\%), and Salyprazone (2.2\%) .Table (6)

The majority $92.2 \%$ would stop the antibiotic if the culture report indicate resistance to that antibiotic and $7.8 \%$ would add another antibiotic to which the microorganism is sensitive.

And $68.9 \%$ would continue using the antibiotic, although the culture showed that microorganism is sensitive to another narrow spectrum antibiotic and only $31.1 \%$ would change to the other narrow spectrum antibiotic.

Table (1): demographic data

\begin{tabular}{|c|c|}
\hline Gender & n (\%) \\
\hline Male & $210(61.9 \%)$ \\
\hline Female & $129(38.1 \%)$ \\
\hline Age & \\
\hline$<1$ & $17(5 \%)$ \\
\hline $1-15$ & $10(2.9 \%)$ \\
\hline $16-30$ & $15(4.4 \%)$ \\
\hline $31-50$ & $77(22.7 \%)$ \\
\hline $51-70$ & $175(51.6 \%)$ \\
\hline $71-98$ & $45(13.3 \%)$ \\
\hline Total & $339(100 \%)$ \\
\hline
\end{tabular}


Journal of Environmental Sciences (JES)

Institute of Environmental Studies and Research, Ain Shams University

Hussein Nourhan et al.

Table (2): Bacterial distribution

\begin{tabular}{|c|c|c|c|c|c|c|c|c|c|c|}
\hline 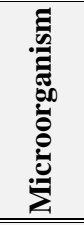 & $\begin{array}{l}\text { Urine } \\
\mathrm{N} \\
(\%)\end{array}$ & $\begin{array}{l}\text { Spu } \\
\text { tum }\end{array}$ & $\begin{array}{l}\text { Pus } \\
\mathrm{N}(\%)\end{array}$ & $\begin{array}{l}\text { Blood } \\
\mathrm{N}(\%)\end{array}$ & $\begin{array}{l}\begin{array}{c}\text { Dra } \\
\text { in }\end{array} \\
\mathrm{N}(\% \\
\mathrm{P} \\
\end{array}$ & $\begin{array}{l}\text { Wo } \\
\text { und }\end{array}$ & $\begin{array}{l}\text { Cen } \\
\text { tral } \\
\text { line } \\
\mathrm{N}( \\
\%) \\
\end{array}$ & $\begin{array}{l}\text { Stool } \\
\mathrm{N}(\%)\end{array}$ & $\begin{array}{c}\text { Endot } \\
\text { rachea } \\
1 \\
\mathrm{~N}(\%)\end{array}$ & $\begin{array}{c}\begin{array}{c}\text { Coun } \\
\mathbf{t}\end{array} \\
(\%)\end{array}$ \\
\hline 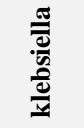 & $\begin{array}{r}27( \\
32.1 \\
4 \%)\end{array}$ & $\begin{array}{l}18( \\
29 \\
\%)\end{array}$ & $\begin{array}{c}14( \\
24.1 \\
\%)\end{array}$ & $\begin{array}{l}5(1 \\
0.6 \\
\%)\end{array}$ & $\begin{array}{r}6(2 \\
0 \%)\end{array}$ & $\begin{array}{c}10( \\
34.4 \\
\%)\end{array}$ & $\begin{array}{l}4(3 \\
3.3 \\
\%)\end{array}$ & $\begin{array}{r}5(5 \\
0 \%)\end{array}$ & $\begin{array}{l}3(4 \\
2.8 \\
\%)\end{array}$ & $\begin{array}{c}92( \\
27.1 \\
\%)\end{array}$ \\
\hline 豙 & $\begin{array}{l}25(29 \\
.7 \%)\end{array}$ & $\begin{array}{c}6(9.6 \\
\%)\end{array}$ & $\begin{array}{c}12(2 \\
0.6 \%)\end{array}$ & $\begin{array}{c}4(8.5 \\
\%)\end{array}$ & $\begin{array}{l}6(20 \\
\%)\end{array}$ & $\begin{array}{l}4(13 . \\
7 \%)\end{array}$ & 0 & $\begin{array}{l}3(30 \\
\%)\end{array}$ & 0 & $\begin{array}{l}60(17 \\
.7 \%)\end{array}$ \\
\hline 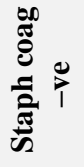 & $\begin{array}{l}4(4 . \\
7 \%)\end{array}$ & $\begin{array}{c}3(4 \\
.8 \% \\
)\end{array}$ & $\begin{array}{c}5(8 \\
.6 \% \\
)\end{array}$ & $\begin{array}{c}25( \\
53.1 \\
\%)\end{array}$ & 0 & $\begin{array}{c}2(6 \\
.8 \% \\
)\end{array}$ & $\begin{array}{l}4(3 \\
3.3 \\
\%)\end{array}$ & 0 & 0 & $\begin{array}{c}43( \\
12.7 \\
\%)\end{array}$ \\
\hline 岕 & $\begin{array}{c}3(3.5 \\
\%)\end{array}$ & $\begin{array}{l}7(11 . \\
2 \%)\end{array}$ & $\begin{array}{r}13(2 \\
2.4 \%)\end{array}$ & $\begin{array}{l}6(12 . \\
7 \%)\end{array}$ & 0 & $\begin{array}{l}4(13 . \\
7 \%)\end{array}$ & 0 & 0 & 0 & $\begin{array}{l}33(9 . \\
7 \%)\end{array}$ \\
\hline 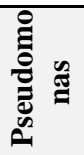 & $\begin{array}{c}7(8.3 \\
\%)\end{array}$ & $\begin{array}{c}6(9.6 \\
\%)\end{array}$ & $\begin{array}{l}7(12 \\
\%)\end{array}$ & 0 & $\begin{array}{c}10(3 \\
3.3 \% \\
)\end{array}$ & $\begin{array}{c}1(3.4 \\
\%)\end{array}$ & $\begin{array}{c}1(8.3 \\
\%)\end{array}$ & 0 & $\begin{array}{l}1(14 . \\
2 \%)\end{array}$ & $\begin{array}{l}33(9 . \\
7 \%)\end{array}$ \\
\hline 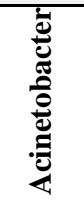 & $\begin{array}{c}3(3.5 \\
\%)\end{array}$ & $\begin{array}{l}18(2 \\
9 \%)\end{array}$ & $\begin{array}{c}2(3.4 \\
\%)\end{array}$ & $\begin{array}{c}2(4.2 \\
\%)\end{array}$ & $\begin{array}{c}2(6.6 \\
\%)\end{array}$ & $\begin{array}{c}2(6.8 \\
\%)\end{array}$ & $\begin{array}{l}2(16 . \\
6 \%)\end{array}$ & 0 & $\begin{array}{l}2(28 . \\
5 \%)\end{array}$ & $\begin{array}{l}33(9 . \\
7 \%)\end{array}$ \\
\hline
\end{tabular}


Journal of Environmental Sciences (JES)

Institute of Environmental Studies and Research, Ain Shams University

Hussein Nourhan et al.

Cont. Table (2): Bacterial distribution

\begin{tabular}{|c|c|c|c|c|c|c|c|c|c|c|}
\hline 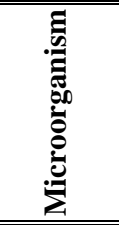 & $\begin{array}{l}\text { Urine } \\
\mathrm{N} \\
(\%)\end{array}$ & $\begin{array}{l}\text { Spu } \\
\text { tum }\end{array}$ & $\begin{array}{c}\text { Pus } \\
\text { N(\%) }\end{array}$ & $\begin{array}{l}\text { Blood } \\
\mathrm{N}(\%)\end{array}$ & $\begin{array}{c}\begin{array}{c}\text { Dra } \\
\text { in }\end{array} \\
\mathrm{N}(\% \\
\\
\end{array}$ & $\begin{array}{l}\text { Wo } \\
\text { und }\end{array}$ & $\begin{array}{l}\text { Cen } \\
\text { tral } \\
\text { line } \\
\mathrm{N}( \\
\%) \\
\end{array}$ & $\begin{array}{l}\text { Stool } \\
\mathrm{N}(\%)\end{array}$ & $\begin{array}{c}\text { Endot } \\
\text { rachea } \\
1 \\
\mathrm{~N}(\%)\end{array}$ & $\begin{array}{c}\begin{array}{c}\text { Coun } \\
\mathbf{t}\end{array} \\
(\%)\end{array}$ \\
\hline 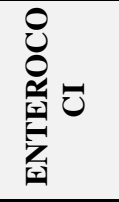 & $\begin{array}{c}4(4.7 \\
\%)\end{array}$ & 0 & $\begin{array}{c}2(3.4 \\
\%)\end{array}$ & $\begin{array}{c}2(4.2 \\
\%)\end{array}$ & $\begin{array}{c}2(6.6 \\
\%)\end{array}$ & $\begin{array}{l}3(10 . \\
3 \%)\end{array}$ & $\begin{array}{c}1(8.3 \\
\%)\end{array}$ & 0 & 0 & $\begin{array}{l}14(4 . \\
1 \%)\end{array}$ \\
\hline$\stackrel{0}{0}$ & $\begin{array}{c}2(2.3 \\
\%)\end{array}$ & $\begin{array}{c}2(3.2 \\
\%)\end{array}$ & 0 & 0 & 0 & $\begin{array}{l}3(10 . \\
3 \%)\end{array}$ & 0 & 0 & 0 & $\begin{array}{c}7(2.1 \\
\%)\end{array}$ \\
\hline 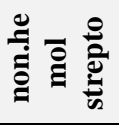 & $\begin{array}{l}5(10 . \\
4 \%)\end{array}$ & 0 & $\begin{array}{l}2(3 \\
.4 \% \\
)\end{array}$ & 0 & $\begin{array}{c}2(6 \\
.6 \% \\
)\end{array}$ & 0 & 0 & 0 & 0 & $\begin{array}{c}9(2 . \\
7 \%)\end{array}$ \\
\hline 竞 & 0 & $\begin{array}{c}2(3 \\
.2 \% \\
)\end{array}$ & $\begin{array}{c}1(1 \\
.7 \% \\
)\end{array}$ & $\begin{array}{c}3(6 \\
.3 \% \\
)\end{array}$ & 0 & 0 & 0 & 0 & $\begin{array}{l}1(1 \\
4.2 \\
\%)\end{array}$ & $\begin{array}{l}7(2 . \\
1 \%)\end{array}$ \\
\hline 苋 & $\begin{array}{l}4(4 . \\
7 \%)\end{array}$ & 0 & 0 & 0 & $\begin{array}{c}2(6 \\
.6 \% \\
)\end{array}$ & 0 & 0 & $\begin{array}{r}2(2 \\
0 \%)\end{array}$ & 0 & $\begin{array}{l}8(2 . \\
4 \%)\end{array}$ \\
\hline हैं & $\begin{array}{c}84( \\
100 \\
\%)\end{array}$ & $\begin{array}{c}62( \\
100 \\
\%)\end{array}$ & $\begin{array}{c}58( \\
100 \\
\%)\end{array}$ & $\begin{array}{c}47( \\
100 \\
\%)\end{array}$ & $\begin{array}{c}30( \\
100 \\
\%)\end{array}$ & $\begin{array}{c}29( \\
100 \\
\%)\end{array}$ & $\begin{array}{c}12( \\
100 \\
\%)\end{array}$ & $\begin{array}{c}10( \\
100 \\
\%)\end{array}$ & 7 & $\begin{array}{c}339 \\
(100 \\
\%)\end{array}$ \\
\hline
\end{tabular}


Hussein Nourhan et al.

Table (3): Sensitivity and resistance pattern of most significant bacteria among antibiotics used:

\begin{tabular}{|c|c|c|c|c|c|c|c|c|}
\hline & \multicolumn{2}{|c|}{ E_coli } & \multicolumn{2}{|c|}{ Acinetobacter } & \multicolumn{2}{|c|}{ Klebsiella } & \multicolumn{2}{|c|}{ Pseudomonas } \\
\hline Antibiotic & $\mathrm{R} \%$ & $\mathrm{~S} \%$ & $\mathrm{R} \%$ & $\mathrm{~S} \%$ & $\mathrm{R} \%$ & S\% & $\mathrm{R} \%$ & S\% \\
\hline Nitrofurantoin & $9.5 \%$ & $\begin{array}{c}90.5 \\
\%\end{array}$ & & & & & & \\
\hline Ceftazidim & $81.5 \%$ & $\begin{array}{c}18.5 \\
\%\end{array}$ & & & $95.8 \%$ & $4.2 \%$ & $\begin{array}{c}93.3 \\
\%\end{array}$ & $6.7 \%$ \\
\hline Amikacin & $16.4 \%$ & $\begin{array}{c}83.6 \\
\%\end{array}$ & $\begin{array}{c}75.9 \\
\%\end{array}$ & $24.1 \%$ & $53 \%$ & $47 \%$ & $\begin{array}{c}65.5 \\
\%\end{array}$ & $34.5 \%$ \\
\hline Impenem & $63 \%$ & $37 \%$ & $\begin{array}{c}88.9 \\
\%\end{array}$ & $11.1 \%$ & $76.4 \%$ & $23.6 \%$ & $\begin{array}{c}77.3 \\
\%\end{array}$ & $22.7 \%$ \\
\hline Tri/sulpha & $82.5 \%$ & $\begin{array}{c}17.5 \\
\%\end{array}$ & $\begin{array}{c}83.3 \\
\%\end{array}$ & 16.7 & $93.6 \%$ & $6.4 \%$ & & \\
\hline Amp/sulbactam & $93 \%$ & $7 \%$ & $90 \%$ & $10 \%$ & $95.5 \%$ & $4.5 \%$ & & \\
\hline Ceftriaxone & $91.5 \%$ & $8.5 \%$ & $\begin{array}{c}100 \\
\%\end{array}$ & --- & $96.5 \%$ & $3.5 \%$ & & \\
\hline Pip/tazo & $46.9 \%$ & $\begin{array}{c}53.1 \\
\%\end{array}$ & $\begin{array}{c}90.9 \\
\%\end{array}$ & $9.1 \%$ & $71.4 \%$ & $28.6 \%$ & $\begin{array}{c}93.3 \\
\%\end{array}$ & $6.7 \%$ \\
\hline Cefipime & $72.7 \%$ & $\begin{array}{c}27.3 \\
\%\end{array}$ & $\begin{array}{c}95.7 \\
\%\end{array}$ & $4.3 \%$ & $91.9 \%$ & $8.1 \%$ & $\begin{array}{c}93.3 \\
\%\end{array}$ & $6.7 \%$ \\
\hline Meropenem & $28.8 \%$ & $\begin{array}{c}66.7 \\
\%\end{array}$ & $\begin{array}{c}77.4 \\
\%\end{array}$ & $22.6 \%$ & $51.9 \%$ & $48.1 \%$ & $\begin{array}{c}75.9 \\
\%\end{array}$ & $24.1 \%$ \\
\hline Amox/clav & $79.5 \%$ & $\begin{array}{c}20.5 \\
\%\end{array}$ & & & $91.5 \%$ & $8.5 \%$ & & \\
\hline Tetracycline & $77.4 \%$ & $\begin{array}{c}22.6 \\
\%\end{array}$ & & & $64.3 \%$ & $35.7 \%$ & & \\
\hline Cefotaxime & $94.3 \%$ & $5.7 \%$ & $\begin{array}{c}100 \\
\%\end{array}$ & --- & $95.5 \%$ & $4.5 \%$ & & \\
\hline Gentamycin & $28.6 \%$ & $\begin{array}{c}71.4 \\
\%\end{array}$ & $\begin{array}{c}81.5 \\
\%\end{array}$ & $18.5 \%$ & $61.3 \%$ & $38.6 \%$ & $63 \%$ & $37 \%$ \\
\hline Ciprofloxacin & $68 \%$ & $32 \%$ & $\begin{array}{c}96.9 \\
\%\end{array}$ & $3.1 \%$ & $81.8 \%$ & $18.2 \%$ & $\begin{array}{c}79.3 \\
\%\end{array}$ & $20.7 \%$ \\
\hline Levofloxacin & $70.2 \%$ & $\begin{array}{c}29.8 \\
\%\end{array}$ & $95 \%$ & $5 \%$ & $71.4 \%$ & $28.6 \%$ & $87 \%$ & $13 \%$ \\
\hline R: Resistant & & $\mathrm{S}:$ & itive & & & & & \\
\hline
\end{tabular}

Vol.(50); Iss.(1); No.(1); Jan.2021 
Journal of Environmental Sciences (JES)

Institute of Environmental Studies and Research, Ain Shams University

Hussein Nourhan et al.

Table (4): Sensitivity and resistance pattern of most significant bacteria among antibiotics used:

\begin{tabular}{|c|c|c|c|c|}
\hline & \multicolumn{2}{|c|}{ SCONs } & \multicolumn{2}{|l|}{ MRSA } \\
\hline Antibiotic & $\mathrm{R} \%$ & $\mathrm{~S} \%$ & $\mathrm{R} \%$ & $\mathrm{~S} \%$ \\
\hline Erythromycin & $76.2 \%$ & $23.8 \%$ & $26.9 \%$ & $73.1 \%$ \\
\hline Cefopid & $100 \%$ & ------ & $100 \%$ & ------ \\
\hline Ceftazidim & $100 \%$ & $\begin{array}{l}------ \\
\end{array}$ & $100 \%$ & ------- \\
\hline Impenem & $100 \%$ & ------ & $100 \%$ & ------ \\
\hline Tri/sulpha & $54.1 \%$ & $45.9 \%$ & $12 \%$ & $88 \%$ \\
\hline Amp/sulbactam & $100 \%$ & ------ & $100 \%$ & ------ \\
\hline ceftriaxone & $100 \%$ & ------ & $100 \%$ & ------ \\
\hline Pip/tazo & $100 \%$ & ------ & $100 \%$ & ------ \\
\hline Cefipime & $100 \%$ & ------ & $100 \%$ & ------ \\
\hline Meropenem & $100 \%$ & ------ & $100 \%$ & ------ \\
\hline Amox/clav & $100 \%$ & ----- & $100 \%$ & ------ \\
\hline Cefotaxime & $100 \%$ & ------ & $100 \%$ & ------ \\
\hline Cefuroxime & $100 \%$ & ----- & $100 \%$ & ------ \\
\hline Teicoplanin & $10 \%$ & $90 \%$ & $3.8 \%$ & $96.2 \%$ \\
\hline Vancomycin & ------ & $100 \%$ & ------ & $100 \%$ \\
\hline Linezolid & ------ & $100 \%$ & ------ & $100 \%$ \\
\hline \multicolumn{5}{|c|}{$\begin{array}{l}\text { R: Resistant S: Sensitive } \quad \text { SCONs: staph coagulase -ve } \\
\text { MRSA: Methicillin Resistant Staphylococcus Aureas }\end{array}$} \\
\hline
\end{tabular}


Table (5): physicians' questionnaire

\begin{tabular}{|c|c|c|c|c|}
\hline \multicolumn{5}{|c|}{ Factors can lead to antibiotic resistance: (can choose more than one answer) } \\
\hline Factors & $\begin{array}{c}\text { very } \\
\text { Important }\end{array}$ & $\begin{array}{l}\text { Moderately } \\
\text { important }\end{array}$ & $\begin{array}{l}\text { Minimally } \\
\text { Important }\end{array}$ & $\begin{array}{l}\text { Don't } \\
\text { know }\end{array}$ \\
\hline widespread use of antibiotic & $\% 98.8$ & $1.2 \%$ & 0 & 0 \\
\hline $\begin{array}{l}\text { overuse of broad-spectrum } \\
\text { antibiotic }\end{array}$ & $100 \%$ & 0 & 0 & 0 \\
\hline inappropriate use of antibiotic & $82.2 \%$ & $17.7 \%$ & 0 & 0 \\
\hline inappropriate choice of antibiotic & $66 \%$ & $28.8 \%$ & $4.4 \%$ & 0 \\
\hline lack of guidelines & $72.2 \%$ & $26.6 \%$ & $1.2 \%$ & 0 \\
\hline patient Demand for antibiotics & $50 \%$ & $34.4 \%$ & $11.1 \%$ & $4.4 \%$ \\
\hline $\begin{array}{|ll|}\text { companies } & \text { promote } \\
\text { antibiotic use }\end{array}$ & 36.6 & $23.3 \%$ & $40 \%$ & 0 \\
\hline inadequate hand washing & 36.6 & $11.1 \%$ & $51.1 \%$ & $1.2 \%$ \\
\hline \multicolumn{5}{|c|}{ Complications related to antibiotic resistance (can choose more than one answer): } \\
\hline Complication & $\begin{array}{l}\text { More } \\
\text { likely }\end{array}$ & $\begin{array}{c}\text { Moderately } \\
\text { likely }\end{array}$ & $\begin{array}{l}\text { Less } \\
\text { likely }\end{array}$ & $\begin{array}{l}\text { Don't } \\
\text { Know }\end{array}$ \\
\hline prolonged treatment & $82.2 \%$ & $17.7 \%$ & 0 & 0 \\
\hline increased cost & $75.5 \%$ & 23.3 & $1.2 \%$ & 0 \\
\hline organ failure & $30 \%$ & $41.1 \%$ & $28.8 \%$ & 0 \\
\hline Death & $7.7 \%$ & $30 \%$ & $54.4 \%$ & $7.7 \%$ \\
\hline \multicolumn{5}{|c|}{ factors are useful in containing resistance : (can choose more than one answer) } \\
\hline Factors & $\begin{array}{c}\text { Very } \\
\text { important }\end{array}$ & Maybe & $\begin{array}{c}\text { Not } \\
\text { important }\end{array}$ & $\begin{array}{l}\text { don't } \\
\text { Know }\end{array}$ \\
\hline educational program & $86.6 \%$ & $13.3 \%$ & 0 & \\
\hline access to lab reports & $68.8 \%$ & $25.5 \%$ & $5.5 \%$ & \\
\hline Consultation & $71.1 \%$ & $27.7 \%$ & $1.2 \%$ & \\
\hline antibiotic guidelines & $71.1 \%$ & $23.3 \%$ & $5.5 \%$ & \\
\hline culture prior to antibiotics use & $77.7 \%$ & $21.1 \%$ & $1.2 \%$ & \\
\hline public educational programs & $62.2 \%$ & $28.8 \%$ & $8.8 \%$ & 0 \\
\hline antibiotic restriction & $61.1 \%$ & $33.3 \%$ & $4.4 \%$ & $1.2 \%$ \\
\hline Antibiotic cycling & $48.8 \%$ & $48.8 \%$ & 0 & $2.2 \%$ \\
\hline
\end{tabular}


Table (6): The most frequent antibiotic prescribed (Empiric antibiotic)

\begin{tabular}{|c|c|c|}
\hline Antibiotic & Count & \% \\
\hline Meropenem & 24 & $26.7 \%$ \\
\hline Ampicillin/sulbactam & 16 & $17.8 \%$ \\
\hline Levofloxacin & 14 & $15.6 \%$ \\
\hline Ceftriaxone & 10 & $11.1 \%$ \\
\hline Ceftazidime & 7 & $7.8 \%$ \\
\hline Ciprofloxacin & 5 & $5.6 \%$ \\
\hline Amikacin & 5 & $5.6 \%$ \\
\hline Dalacin & 4 & $4.4 \%$ \\
\hline Tazocin & 3 & $3.3 \%$ \\
\hline Salyprazone & 2 & $2.2 \%$ \\
\hline Total & 90 & $100.0 \%$ \\
\hline
\end{tabular}

\section{DISCUSSION}

The widespread of MDR pathogens is becoming a serious challenge in public health. Recently, therapeutic options against them have become limited, causing morbidity and mortality in hospitalized patients. Inappropriate use of antibiotics in hospitals and communities has resulted in an increasing resistance to various antibiotics (Awad and Aboud, 2015).

In this study the total number of isolated pathogens was (339) among which Klebsiella was the most common isolate (27.1\%), followed by E_coli (17.7\%), Staph coag -ve (12.7\%) and MRSA, Pseudomonas and Acinetobacter $(9.7 \%)$ for each. Similar to our study results, literature also reports Klebsiella pneumoniae as the most common resistant isolates, followed by Escherichia coli and/or Enterobacter species. (Sodhi, et al., 2020) 14 
Journal of Environmental Sciences (JES)

Institute of Environmental Studies and Research, Ain Shams University

Hussein Nourhan et al.

and also similar to a study conducted at tertiary care hospital in Egypt by (Hassan, et al., 2020).

The distribution of those resistant pathogen mainly found in age range (51-70 years) with (51.6\%) then from (31-50 years) with $(22.7 \%)$ which support that aging is one of the main predisposing risk factors for HAIs with resistant pathogens, this was in agreement with Feng, et al., (2019).

In our study E.coli showed the lowest resistance pattern to most antibiotics specially gentamycin and amikacin unlike A.baumanni \& P. aeruginosa which were highly resistant to the same antibiotics. However the highest sensitivity of E.coli was recorded to nitrofurantoin (90\%) as found in other study by (Aguilar-Santelises, et al., 2020).

Klebsiella which were the Most frequent isolate in our study unlike E.coli showed moderate resistance to meropenem (51.9\%) and amikacin (53 $\%)$ and were highly resistant to ceftriaxone (96.5\%) followed by ceftazidim $(95.8 \%)$ and levofloxacin(71.4\%). Another study at largest hospital's NICU in Minia by Hassuna, et al., (2020) showed higher degree of resistance towards antibiotics with $(100 \%)$ resistance against ceftriaxone, while unlike our study they found none resistant against levofloxacin.

According to the results none of MRSA and SCONs were resistant to linezolid nor vancomycin. However, MRSA which was the dominant bacterial species isolated from pus showed higher sensitivity against teicoplanin, tri/sulpha and erythromycin unlike SCONs which was the dominant bacterial species isolated from blood showed moderately higher 
Journal of Environmental Sciences (JES)

Institute of Environmental Studies and Research, Ain Shams University

Hussein Nourhan et al.

resistance against them which was consistence with other studies byHashemizadeh, et al., (2019) and Nicolosi,D., et al.,(2020).

Antimicrobial stewardship programs (ASPs) have emerged as a strategy to manage or contain the problem of AMR, which considered as a systematic approach to improve the prescribing pattern of antibiotics through a variety of interventions and have been proven to be cost-effective. ASPs should afford educational and effect changes in prescribing behaviors of healthcare providers. (Labricciosa, F. et al., 2018)

In this context, a questionnaire was conducted to analyze the use and prescribing pattern of physicians working at the same departments from which the resistant pathogen were isolated at the same period of time from which isolated pathogens were collected. The total number of physicians who filled the questionnaire were (90) physician of different specialties and years of experience from which $(55.6 \%)$ male and $(44.4 \%)$ female.

In this study, the majority of physicians $(85.6 \%)$ have good knowledge about the antibiotic resistance meaning and the magnitude of that problem which was in agreement with a study conducted at national liver institute in Egypt by Tahoon, et al., (2020) who found that (85\%) of physician were familiar with antibiotic resistance. another study by Tegagn et al., (2017) found that $81.3 \%$ of healthcare professionals were familiar with antibiotic resistance meaning.

According to the conducted survey Overuse of broad spectrum antibiotic considered to be the most Important factor that lead to antibiotic resistant 
Journal of Environmental Sciences (JES)

Institute of Environmental Studies and Research, Ain Shams University

Hussein Nourhan et al.

(100\%)followed by Widespread use of antibiotic (98.9\%) and inappropriate use of antibiotic (82.2\%) These results are close to previous study from Italy Napolitano F, et al., (2013) also showed that wide spread use of antibiotics is the most important cause of this problem.

Most Physicians thought that prolonged Treatment (74\%) and increased cost $(68 \%)$ were the most frequent complications related to ANTIBIOTIC resistance and Death is the least complication (7\%) which was similar to another survey by Nichol, , et al,. (2018) at a tertiary care institution in the Caribbean.

The majority of physicians (78\%) considered periodic Educational programs to be the most useful factor in containing the antibiotic resistance problem as a tool to improve antibiotic prescription pattern of them. Furthermore, the majority considered taking cultures prior to ANTIBIOTIC choice $(70 \%)$ as other important factor against the spread of MDR bacteria. Antibiotic cycling considered to be the least useful factor with (44\%) which is correlate with a study in UAE by John, et al., (2012)and other study in Khartoum by Kheder .,(2013).

This results shows that there is a need to follow infection control measures and to increase the restrictions of antibiotic use in the current antibiotic policy to slow down the emergence and spread of antibiotic resistant bacteria in the health care setting. 
Journal of Environmental Sciences (JES)

Institute of Environmental Studies and Research, Ain Shams University

Hussein Nourhan et al.

\section{CONCLUSION}

Infections can be transferred between and within hospitals, facilitated by sub-optimal infection prevention and control measures, as well as between the community and healthcare facilities.

Hence, the importance of cooperation between clinical and microbiology laboratory staff in determining antibiotic resistance profiles and implementation of effective antibiotic stewardship programs in controlling selection and use of antibiotics.

\section{REFERENCES}

Aguilar-Santelises, M., Castillo-Vera, J., Gonzalez-Molina, R. et al. (2020): Clinical isolates of Escherichia coli are resistant both to antibiotics and organotin compounds. Folia Microbiol 65, 87-94.

Atif, M, Azeem, M., Saqib, A.; scahell,S. (2017). Investigation of antimicrobial use at a tertiary care hospital in Southern Punjab, Pakistan using WHO methodology. Antimicrob Resist Infect Control 6, 41.

Awad AI, Aboud EA (2015) Knowledge, Attitude and Practice towards Antibiotic Use among the Public in Kuwait. PLoS ONE 10(2): e0117910.

Centers for Disease Control and Prevention, Office of Infectious Disease Antibiotic resistance threats in the United States, 2013. Apr, 2013.

Feng,D,; zhou, Y; Zou,X; et al. (2019). Factors influencing mortality in hospital-acquired pneumonia caused by Gram-negative bacteria in China. Journal of infection and public health, 12(5), 630-633. 
Journal of Environmental Sciences (JES)

Institute of Environmental Studies and Research, Ain Shams University

Hussein Nourhan et al.

Gould, I. M., \& BAL, A. M. (2013). New antibiotic agents in the pipeline and how they can help overcome microbial resistance. Virulence, 4(2), 185-191.

Hashemizadeh, Z., Hadi, N., Mohebi, S., Kalantar-Neyestanaki, D., \& Bazargani, A. (2019). Characterization of SCCmec, spa types and Multi Drug Resistant of methicillin-resistant Staphylococcus aureus isolates among inpatients and outpatients in a referral hospital in Shiraz, Iran. BMC research notes, 12(1), 614.

Hassan, R, El-Gilany, A, Abd-elaal, MA, et al., (2020). An overview of healthcare-associated infections in a tertiary care hospital in Egypt. Infection Prevention in Practice Volume 2, Issue 3, 100059 .

Hernando-Amado, S, Sanz-Garcia, F, Blanco, P,and Martinez JL. (2017). Fitness costs associated with the acquisition of antibiotic resistance .Essays in biochemistry 61 (1), 37-48.

Hssouna, N.A, AbdelAziz RA; Zakaria A and Abdelhakeem M (2020) Extensively-Drug Resistant Klebsiella pneumoniae Recovered From Neonatal Sepsis Cases From a Major NICU in Egypt. Front. Microbiol. 11:1375. doi: 10.3389/fmicb.2020.01375

John, L.J., Arifulla, M., Cheriathu, J.J. et al. (2012). Reporting of adverse drug reactions: an exploratory study among nurses in a teaching hospital, Ajman, United Arab Emirates. DARU J Pharm Sci 20, 44.

Kheder SI. (2013). Physcians knowledge and perception of antimicrobial resistance: a survey in Khartoum Stata Hospital settings. Br J Pharmaceut Res. (3):347-362.

Labi,AK, Obeng-Nkrumah ,N ; Nartey, ET; et al., (2018).Antibiotic use in a tertiary healthcare facility in Ghana: a point prevalence survey . Antimicrob Resist Infect Control (7), 15. 
Labricciosa, F.M., Sartelli, M., Correia, S. et al. (2018).Emergency surgeons' perceptions and attitudes towards antibiotic prescribing and resistance: a worldwide cross-sectional survey. World J Emerg Surg (13), 27.

Laxminarayan, R. (2014). Antibiotic effectiveness: Balancing conservation against innovation. Science (New York, N.Y.). 345. 1299-301. 10.1126/science. 1254163 .

Napolitano F, Izzo, M. T., Di Giuseppe, G., \& Angelillo, I. F. (2013). Public knowledge, attitudes, and experience regarding the use of antibiotics in Italy. PloS one, 8(12), e84177.

Nichol,A, Tennant, I., White, L. et al. (2018).The knowledge, attitudes and practices of doctors regarding antibiotic resistance at a tertiary care institution in the Caribbean. Antimicrob Resist Infect Control (7), 23.

Nicolosi, D, et al. (2020). Antimicrobial Resistance Profiling of CoagulaseNegative Staphylococci in a Referral Center in South Italy: A Surveillance Study. The Open Microbiology Journal, Volume 14, 91-97.

Sodhi,K, Mittal, V., Arya, M., Kumar, M., Phillips, A., \& Kajla, B. (2020). Pattern of colistin resistance in Klebsiella isolates in an Intensive Care Unit of a tertiary care hospital in India. Journal of infection and public health, 13(7), 1018-1021.

Tahoon, M.A., Khalil, M.M., Hammad, E. et al. (2020). The effect of educational intervention on healthcare providers' knowledge, attitude, \& practice towards antimicrobial stewardship program at, National Liver Institute, Egypt. Egypt Liver Journal (10), 5.

Tegagn GT, Yadesa TM, Ahmed Y. (2017). Knowledge, attitudes and practices of healthcare professionals towards antimicrobial stewardship and their predictors in Fitche Hospital. J Bioanalysis Biomed 9(2):91-97. 
Tyers, M and Wright, G.D. (2019). Drug combinations: a strategy to extend the life of antibiotics in the 21 st century. Nat Rev Microbiol 17, 141-155.

Ventola,CL. (2015). The antibiotic resistance crisis: part 1: causes and threats. P \& T: a peer-reviewed journal for formulary management, 40(4), 277-283.

World health organization (WHO) ,antibiotic resistance , 5 feb 2018 at: https://www.who.int/news-room/fact-sheets/detail/antibioticresistance

Zulu A, Matafwali SK, Banda M, Mudenda S. (2020). Assessment of knowledge, attitude and practices on antibiotic resistance among undergraduate medical students in the school of medicine at the University of Zambia. Int J Basic Clin Pharmacol (9):263-70.

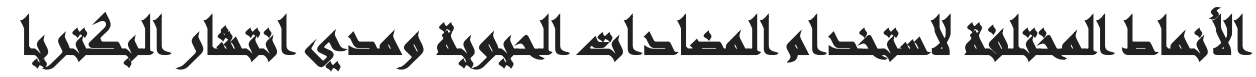

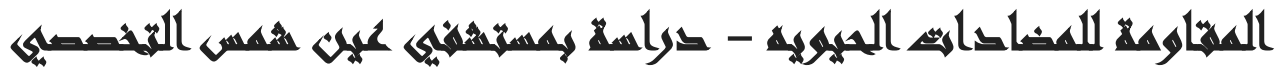

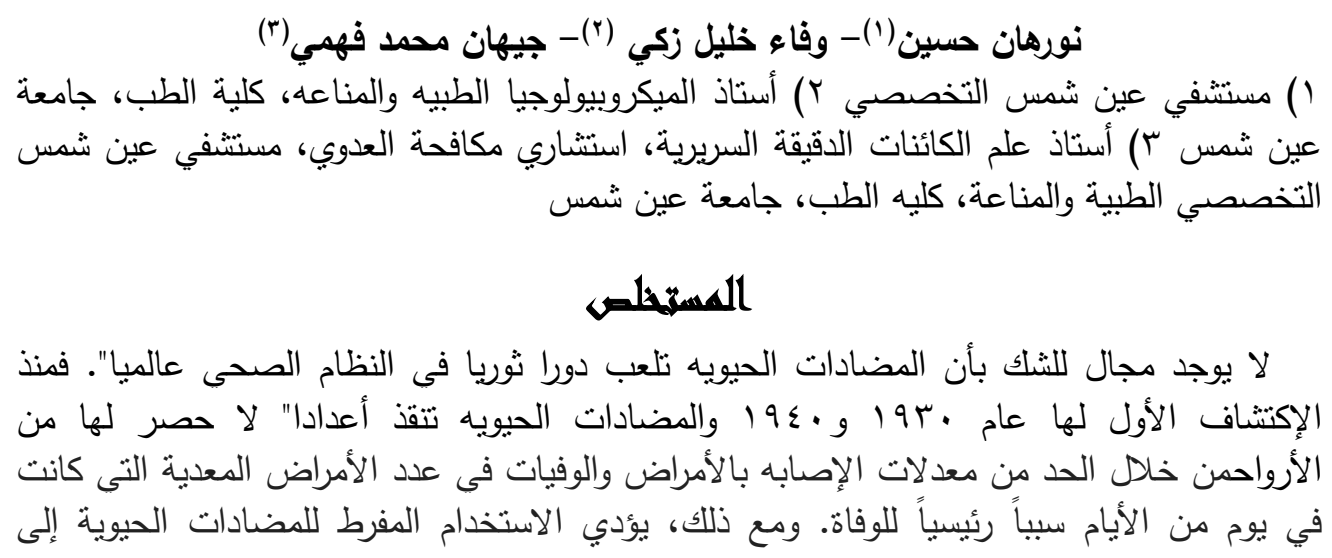


Journal of Environmental Sciences (JES)

Institute of Environmental Studies and Research, Ain Shams University

Hussein Nourhan et al.

تطوير وانتشار البكتبريا المقاومة للمضادات الحيويه، والتي تُعتبر حاليًا أزمة عالمية للصحة العامة

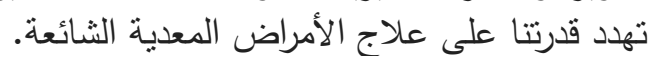

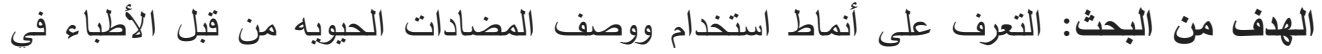

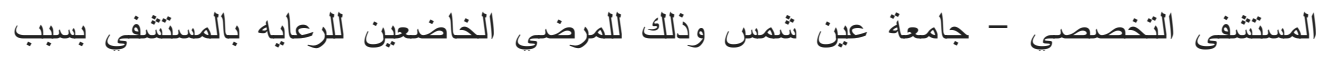

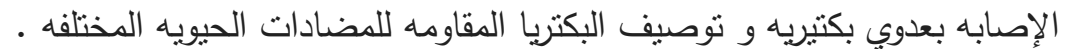

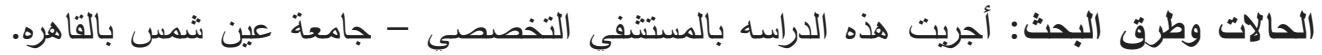

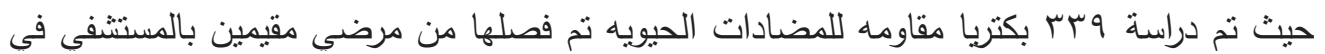

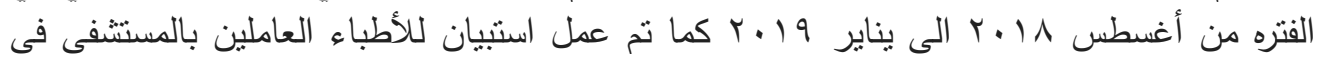

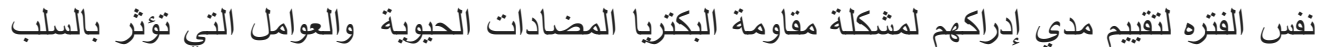
وتزيد من فرص حدوث مقاومه بكتيريه.

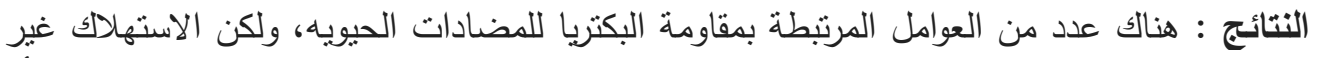

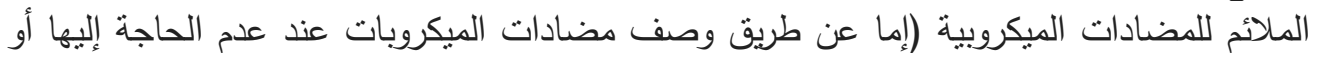

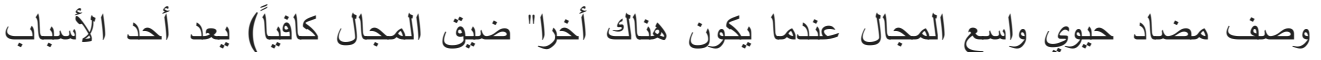
الرئيسيه.

الخلاصه: لابد من مراقبة استخدام المضادات الحيوية كإستراتيجية لتحسين استخدام المضادات

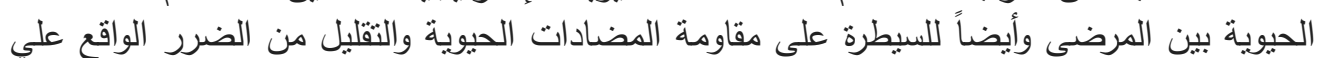

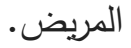
الكلمات الرئيسيه : البكتريا المقاومه للمضادات الحيويه، أنماط وصف المضادات الحيويه. 